\title{
A FAST COMMUNITY BASED ALGORITHM FOR GENERATING WEB CRAWLER SEEDS SET
}

\author{
Shervin Daneshpajouh, Mojtaba Mohammadi Nasiri ${ }^{1}$ \\ Computer Engineering Department, Sharif University of Technology, Tehran, Iran \\ daneshpajouh@ce.sharif.edu,m_mohammadi@ce.sharif.edu \\ Mohammad Ghodsi ${ }^{2}$ \\ Computer Engineering Department, Sharif University of Technology, Tehran, Iran \\ School of Computer Science, Institute for Studies in Theoretical Physics and Mathematics, Tehran, Iran \\ ghodsi@sharif.edu
}

\begin{abstract}
Keywords: Crawling, Communities, Seed Quality Metric, Crawl Quality Metric, HITS, Web Graph, Hyperlink Analysis.

Abstract: In this paper, we present a new and fast algorithm for generating the seeds set for web crawlers. A typical crawler normally starts from a fixed set like DMOZ links, and then continues crawling from URLs which are found in these web pages. Crawlers are supposed to download more good pages in less iteration. Crawled pages are good if they have high PageRanks and are from different communities. In this paper, we present a new algorithm with running time $O(n)$ for generating crawler's seeds set based on HITS algorithm. A crawler can download qualified web pages, from different communities, starting from generated seeds set using our algorithm in less iteration.
\end{abstract}

\section{INTRODUCTION}

A major question a crawler has to face is which pages are to be retrieved so as to have the "most suitable" pages in a collection [1]. Crawlers normally retrieve a limited number of pages. In this regard, the question is how fast a crawler collects the "most suitable" pages. A unique solution to this question is not likely to exist. In what follows, we try to answer this question.

Different algorithms with different metrics have been suggested to lead a crawl towards high quality pages [2,3]. In [2] Cho, Garcia-Molina, and Page suggested using connectivity-based metrics to do so. To direct a crawl, they have used different ordering metrics: breadth-first, backlink count, PageRank, and random. They have revealed that performing a crawl in breadth-first order works nearly well if "most suitable" pages are defined to be pages with high PageRanks.

Najork and Wiener extended the results of Cho et al. They examined the average page quality over the time of pages downloaded during a web crawl of
328 million unique pages and showed that traversing the web graph in breadth-first search order is a good crawling strategy[3].

Based on Henzinger's work [1] better understanding of graph structure might lead to a more efficient way to crawl the web. In this paper we use this idea to develop our algorithm. First, we define the "most suitable" pages and then we show how a crawler can retrieve them. We use three metrics to measure the quality of a page.

In this paper, we present a new fast algorithm for extracting seeds set from previously crawled pages. Using offered metrics, we show that by starting from extracted seeds suggested by our algorithm, a crawler will quickly collect the most suitable pages from different communities.

We have studied different community extraction algorithms: PageRank[4], Trawling[8], HITS, and Network flow base community discovery[5,9]. After analysis, we decided to use HITS-Ranking without keyword search in our algorithm for community discovery and collecting seeds set. We have found that bipartite cores are useful for selecting seeds set.

\footnotetext{
${ }^{1}$ This author's work was in part supported by a grant from ITRC (N. T-500-1895-86-2-17.)

${ }^{2}$ This author's work was in part supported by a grant from IPM (N. CS2386-2-01.)
} 
Bipartite cores contain Hub and Authority pages. Since we are interested in having Authority pages in our crawl, we would need to start crawling from Hub pages. Hubs are durable pages, so we can rely upon them for crawling.

The main idea in our method is to use HITSRanking on the whole graph for extracting the most important bipartite cores. We offer two bipartite core extraction algorithms.

We have compared the results of the crawls starting from extracted seeds set produced by our algorithm, with crawls starting random nodes. Our experiments show that the crawl starting from seeds set identified by our algorithm finds the most suitable pages of web very faster than a random crawler did.

According to our knowledge, this is the first seeds extraction algorithm that is able to identify and extract seeds from different web communities. Low running time is crucial in working with large size web data. The running time of proposed algorithm is $O(n)$. Low running time with community base properties makes this algorithm unique in comparison with previous algorithms.

The remainder of this paper proceeds as follows: in Section 2, we present our algorithm for discovering seeds set in a large web graph and compute the complexity of proposed algorithm; in Section 3, we discuss the results of running and evaluating this algorithm on $18 \mathrm{M}$ and $39 \mathrm{M}$ node graphs; Section 4 contains conclusion and future works.

\section{ALGORITHM FOR DISCOVERING SEEDS SET IN LARGE WEB GRAPH}

In this section, we present our algorithm to discover seeds sets from web graph.

A crawler normally does not crawl the entire web. Instead, it continues to retrieve a limited number of pages. Crawlers are expected to collect the "most suitable" pages of web rapidly. We defined "most suitable" pages of web as those pages with high Page Rank. In terms of HITS algorithm they are called Authority pages. The difference is that HITS algorithm finds the authority pages relating to keywords but PageRank shows the importance of a page in the whole web. As well, we know that good hubs link to good authorities. If we are able to extract good hubs from a web graph and different communities, we will be able to download good authorities that have high PageRank of different communities.

\subsection{Iterative HITS-Ranking \& Pruning}

We assume that we have a web graph of crawled web pages. The goal is to extract seeds set from this graph so that a crawler can collect the most important pages of the web in less iteration. To do this we run HITS-ranking algorithm on this graph. This is the second step of HITS algorithm. In the first step, it searches the keywords in an index-based search engine. For our purpose, we ignore this step and only run the ranking step on the whole graph. In this way, bipartite cores with high Hub and Authority rank will become visible in the graph. Then we select the most highly ranked bipartite core using two algorithms. We suggest, extracting seeds with fixed size, and extracting seeds with fixed density; we remove this sub-graph from the graph, repeat ranking, seed extraction, and sub-graph removal steps up to a point that we have enough seeds set.

A question that may arise is why we need to run HITS-ranking again when repeating these steps. Isn't one time ranking enough for whole steps? The answer is: removing bipartite core in each step modifies the web-graph structure we are working on. In fact, re-ranking changes the hub and authority ranks of bipartite cores. Removing high-ranked bipartite core and re-ranking web-graph drive, bipartite cores appeared to be from different communities. Thus, a crawler will be able to download pages from different communities starting from these seeds. We have experimented our algorithm using web-graph of UK 2002 containing 18M nodes and 298M edges, and UK 2005 containing 39M nodes and 936M edges [6, 7]. Our experiments prove that extracted bipartite cores have a reasonable distance from each other.

The other question that may arise is that if a crawler starts from seeds resulted from our algorithm, why would the results of crawl lead to the most suitable pages. The answer is: in iterations of algorithm, we select and extract high-ranked bipartite cores from the web-graph. Extracted bipartite cores have high hub or authority ranks. It is expected that pages with high hub-rank link to pages with high PageRank. Our experiments prove the correctness of this hypothesis.

\subsection{Extracting Seeds with Fixed Size}


The Extract-Bipartite-Cores-with-Fixed-Size procedure, as it's name indicates, extracts one bipartite sub-graph with highest hub and authority ranks with predetermined size given as an input. Algorithm is given a directed graph $G$, BipartiteCoreSize, NewMemberCount and $h$, and $a$ vectors. BipartiteCoreSize specifies the desired size of bipartite core we like to be extracted. NewMemberCount indicates in each iteration of algorithm how many hub or authority nodes should be added to the hub or authority sets; $h$ and $a$ vectors are hub and authority ranks of nodes in the input graph $G$.

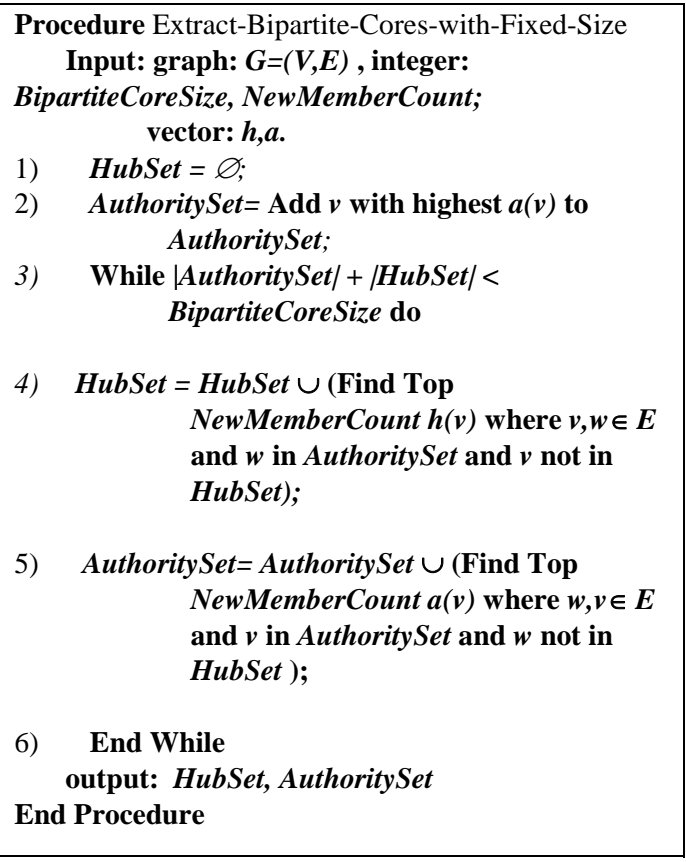

Figure 1. Extracting Bipartite Cores with Fixed Size

In the initial steps, the Algorithm sets HubSet to empty and adds the node with highest authority rank to AuthoritySet. While the sum of AuthoritySet size and HubSet size is less than BipartiteCoreSize, it continues to find new hubs and authorities regarding the NewMemberCount and adds them to the related set. We use this procedure when we like to extract bipartite sub-graph with fixed size. Figure 1 shows the details of Extract-Bipartite-Cores-with-FixedSize procedure. In Figure 2 we show the steps of bipartite sub-graph creation with NewMemberCount equal to 1. An interesting result we have found in our experiments is that at the very first steps, all the hubs have links to all authorities which is a complete bipartite sub-graph. This leaded us to suggest an extraction algorithm with a density factor that is described in the following subsection.

\subsection{Extracting Seeds with Fixed Cover-Density}

The Extract-Bipartite-Cores-with-FixedCoverDensity procedure, as it's name indicates, extracts one bipartite sub-graph with highest hub and authority ranks in a way that the sub-graph has the desired cover-density function. A directed graph $G$, CoverDenstity, $h$ and $a$ vectors are given to the algorithm.

We define Cover-Density as follows:

$$
100 * \frac{\mid E(\text { HubSet, AuthoritySet }) \mid}{\mid \text { HubSet }|| \text { AuthoritySet } \mid}
$$

This measure shows how many nodes in the authority set are covered by nodes in hub set. If the bipartite sub-graph is a complete bipartite sub-graph, this measure will be equal to 100 . Therefore, if we intend to extract complete bipartite sub-graph we set CoverDenstity to $100 . h$ and $a$ vectors are hub and authority ranks of nodes in the input graph $G$.

In initial steps, Algorithm sets HubSet to empty set and adds the node with highest authority rank to AuthoritySet. In addition, it sets CoverDensityCur to 100.

While CoverDensityCur is bigger than or equal to input CoverDensity, procedure continues to find new hubs and authorities. This algorithm adds only one new node to the sets at each iteration of the algorithm. Remember that in Extract-BipartiteCores-with-Fixed-Size we could adjust the count of new members. Here, we do not have such a variable. This is because of the fact that we like to have a precise cover density here. In other words, if we increase the number of new nodes to more than 1 , this might cause the reduction of the accuracy of desired cover density.

We use this procedure when we like to extract bipartite sub-graph with desired density between hubs and authorities. Figure 3 shows the details of the

Extract-Bipartite-Cores-with-FixedCoverDensity procedure.

\subsection{Putting It All Together}

Up to now, we have presented algorithms for HITSRanking and bipartite core extraction based on hub and authority ranks. Our goal is to extract a set of 
desired number of seeds to crawl and download pages from different web communities with high PageRank in less iteration. We use the proposed algorithms to achieve this goal. We assume that we have a web graph of crawled web pages. Then we run HITS-Ranking algorithm on the whole graph and use one of the bipartite core extraction algorithms we have presented. Then we select arbitrarily one of the nodes in the extracted hub set and add it to our seeds set. Finally, we remove the extracted core from the input graph and repeat these steps until we find the ideal number of seeds. (a)

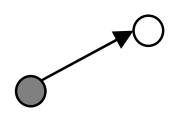

(c)

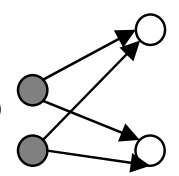

(b)

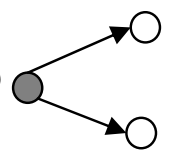

(d)

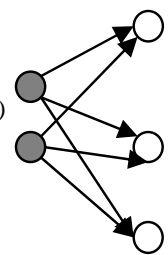

Figure 2. Steps of bipartite sub-graph creation with NewMemberCount equal to 1 . Hub nodes are shown in grey and authority nodes are shown in white. (a) shows the sub-graph after adding the highest authority rank node and adding hub with highest rank that refer to this authority node. In (b), the next authority with highest rank which was not added previously in authority set and is linked by the only node in the hub set is added. In (c), the second hub node with highest hub rank which was not already in the hub set and linked to one of the nodes in authority set is added. In (d) resulted sub-graph after 4 steps is shown.

We can use one of these two bipartite core extraction algorithms that we have proposed: Extract-Bipartite-Cores-with-Fixed-Size, ExtractBipartite-Cores-with-Fixed-CoverDensity. If we wish bipartite cores to have a fixed size we use the first algorithm and if we are looking for bipartite cores having desired cover density, then we use the second algorithm. For example, if we like the density of bipartite cores to be complete we should use the second algorithm.

We have experimented both of these algorithms. As we cannot guess the suitable size of a web community, we use the second method. The second method can calculate the density of links between hubs and authorities. If we have a complete bipartite core then we are sure that all the authority pages are from the same community. By decreasing the CoverDensity measure, we decrease the degree of relationship between authority pages. Because the second method is more reliable than the first one, in this paper we only present experimental results achieved from using Extract-Bipartite-Cores-withFixed-CoverDensity. Figure 4 shows the seeds extraction algorithm we have used in our experiments in this paper.

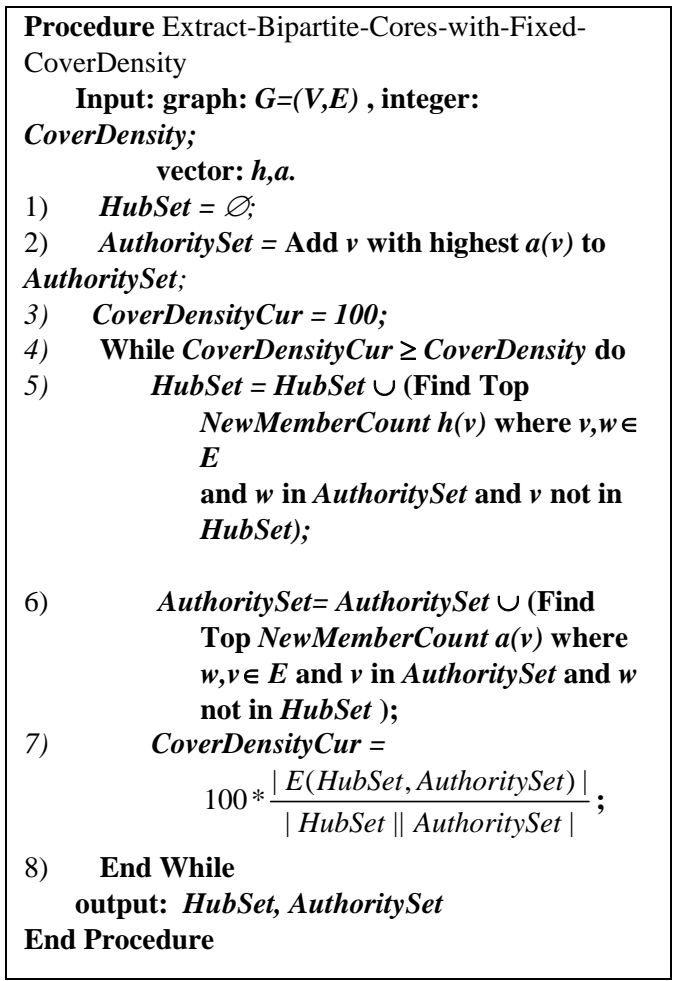

Figure 3. Extracting Bipartite Cores with Fixed Density

The Extract-Seeds algorithm receives a directed graph $G$ and SeedCount as input. At the initial step, algorithm sets SeedSet to empty. While the size of SeedSet is less than SeedCount, the algorithm keeps running. In the first line of While section, algorithm calls HITS-Ranking procedure with $G$ as the input graph and 60 as HITSIterationCount. Kleinberg's work shows that HITSIterationCount equal to 20, is enough for convergence of hub and authority ranks in a small sub-graph [7]. We have found experimentally that a number of more than 50 is enough for convergence of hub and authority ranks with the dataset we use. HITS-Ranking algorithm returns two vectors, $h$ and $a$, containing result of hub and authority ranks of all nodes in graph $G$. In the next line algorithm calls Extracting-Bipartite-Coreswith-Fixed-CoverDensity with $G$ as input graph, 100 as cover density value, and $h$ and $a$ as hub and authority vectors. This function finds complete 
bipartite cores in the input graph and returns complete bipartite nodes in HubSet and AuthoritySet. In the next line, a node randomly is selected from hub set and is added to the SeedSet. Now algorithm removes the hub and authority nodes and their edges from graph $G$. The removal step helps us to find seeds from different communities.

\begin{tabular}{|c|c|}
\hline \\
\hline \multicolumn{2}{|c|}{ Input: graph: $G=(V, E)$, integer: SeedCount; } \\
\hline 1) & SeedSet $=\varnothing$ \\
\hline 2) & While $\mid$ SeedSet $\mid<$ SeedCount do \\
\hline 3) & $\boldsymbol{h}, \boldsymbol{a}=$ HITS-Ranking $(\boldsymbol{G}, \mathbf{6 0})$; \\
\hline 4) & HubSet, AuthoritySet = Extracting- \\
\hline & Bipartite-Cores-with-Fixed- \\
\hline & CoverDensity $(G, 100, h, a)$; \\
\hline 5) & $\begin{array}{l}\text { SeedsSet }=\text { SeedsSet } \cup \text { Select } \text { a node } \\
\text { arbitrarily from HubSet; }\end{array}$ \\
\hline 6) & For all $v$ in HubSet do \\
\hline 7) & Remove $v$ and all $E(v)$ from $G$; \\
\hline 8) & End For \\
\hline 9) & For all $v$ in AuthoritySet do \\
\hline 10) & Remove $v$ and all $E(v)$ from $G$ \\
\hline 11) & End For \\
\hline 12) & End While \\
\hline & output: SeedsSet \\
\hline Enc & Procedure \\
\hline
\end{tabular}

Figure 4. Seeds Extraction Algorithm

\subsection{Complexity of Proposed Seeds Extraction Algorithm}

The running time of Seeds-ExtractionAlgorithm, (Figure 4), is $O(n)$, where $n$ is the number of nodes in the input graph.

The While loop of lines 2-12 is executed at most |SeedCount| times. The work of line 4 is done in $O(n)$. Because the complexity of HITS-Ranking is equal to $\Theta\left(K^{*} 2 * L^{*} n\right)$ where $\mathrm{K}$ is |HitsIterationCount|, $\mathrm{L}$ the average number of neighborhoods of a node and $n$ is the number of nodes in the input graph. This complexity is multiplied by 2 because there are two steps for this kind of computation, one for hub vector and the other for authority vector. In addition, the normalization steps can be done in $\Theta(3 n)$. So, the complexity of HITS-Ranking is $O(n)$.

The running time of Extracting-Bipartite-Coreswith-Fixed-CoverDensity in line 4 is $O(n)$. The While loop of lines $4-8$, in figure 3 , is executed at most $\mid$ HubSet + AuthoritySet $\mid$ times which can be viewed as a constant number k. Finding and adding a distinct hub node with highest rank to hub set, in line 5, takes $\Theta\left(k^{*} n\right)$. Finding and adding a distinct authority node with highest rank to authority set, in line 6, takes $\Theta\left(k^{*} n\right)$. So, the running time of Extracting-Bipartite-Cores-with-Fixed-

CoverDensity is at most $O(n)$.

The removal steps of lines 6-11, in Figure 4, takes $O(n)$ for removing identified hubs and authorities.

Therefore, the total running time of Seed-Extraction Algorithm is $O\left(\mid\right.$ SeedCount $\left.{ }^{*} n\right)$, which is equal to $O(n)$.

\section{EXPERIMENTAL RESULTS}

In this section, we apply our proposed algorithm, to find seeds set from previously crawled pages. Then, we start a crawl using extracted seeds on the same graph to evaluate the result. To show how applying algorithm on old data can provide good seeds for a new crawl, we start the crawl on a newer graph using seeds set extracted from a previous crawl.

\subsection{Data Sets}

The laboratory for Web Algorithmics at the University of Milan provides different web graph data sets [10]. In our experiments, we have used UK-2002 and UK-2005 web graph data sets provided by this laboratory. These data sets are compressed using WebGraph library. WebGraph is a framework for studying the web graph [11]. It provides simple ways to manage very large graphs, exploiting modern compression techniques. With WebGraph, we can access and analyze a very large web graph on a PC.

\subsubsection{UK-2002}

This data set has been obtained from a 2002 crawl of the .uk domain performed by UbiCrawler in 2002 [12]. The graph contains 18,520,486 nodes and 298,113,762 links.

\subsubsection{UK-2005}

This data set has been obtained from a 2005 crawl of the .uk domain performed by UbiCrawler in 2005. The crawl was very shallow, and aimed at gathering a large number of hosts, but from each host a small number of pages. This graph contains 39.459.935 nodes and 936,364,282 links. 


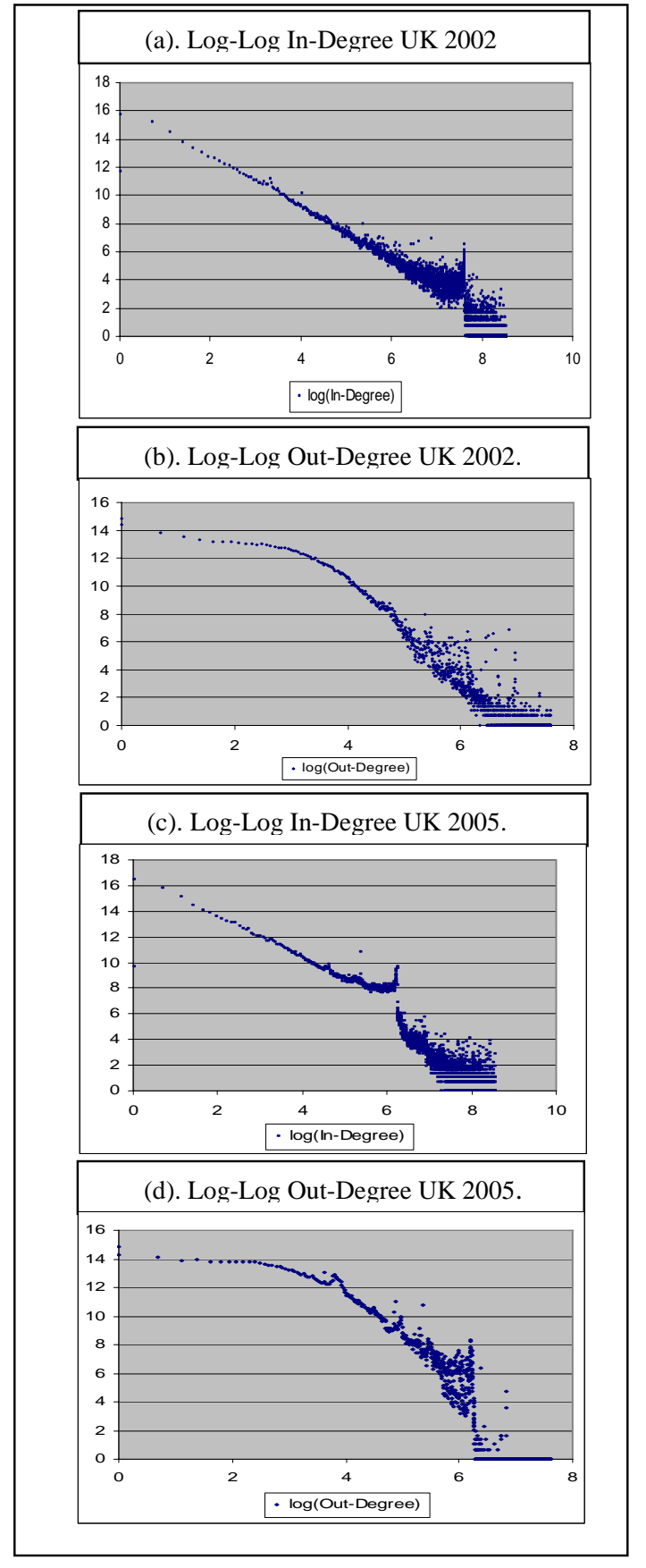

Figure 5. diagram of Log-Log In-Degree and Out-Degree of UK 2002 and UK 2005.

\subsection{Data Set Characteristics}

\subsubsection{Degree Distribution}

We had investigated the degree distribution of UK2002 and UK-2005. Figure 7.a and 7.b show Indegree and Out-degree distribution for UK-2002 in log-log form. Figure 7.c and 7.d show In-degree and Out-degree distribution for UK-2005 in log-log form. The results show that the In-degree and Outdegree distribution are power laws in these two datasets.

\subsubsection{Diameter}

The diameter of a web-graph is defined as the length of shortest path from $\mathrm{u}$ to $\mathrm{v}$, averaged over all ordered pairs $(u, v)$ [13]. Of course, we omit the infinite distance between pairs that there is not a path between them. This is called average connected distance in [6]. We estimated this measure on UK2002 and UK-2005 data sets through experiments. Table 1 shows the estimated diameter of these data sets together with the number of nodes and edges. We use the resulted diameter to evaluate the distances between extracted bipartite cores resulting from our method.

Table 1. UK 2002 and 2005 Data Sets information before pruning.

\begin{tabular}{|c|c|c|c|}
\hline Data Set & Nodes & Edges & $\begin{array}{c}\text { Diameter } \\
\text { Estimate }\end{array}$ \\
\hline UK-2002 & $18,520,486$ & $298,113,762$ & 14.9 \\
\hline UK-2005 & 39.459 .935 & $936,364,282$ & 15.7 \\
\hline
\end{tabular}

Table 2. UK 2002 and 2005 Data Sets information after pruning.

\begin{tabular}{|c|c|c|}
\hline Data Set & Nodes & Edges \\
\hline UK-2002 & $18,520,486$ & $22,720,534$ \\
\hline UK-2005 & 39.459 .935 & $183,874,700$ \\
\hline
\end{tabular}

\subsection{Date Preparation}

\subsubsection{Pruning}

Most of the links between pages in a site are for navigational purposes. These links may distort the result of presented algorithm. The result of the HITS-Ranking algorithm on this un-pruned graph will result in hub and authority pages to be found in a site. To eliminate this effect we remove all links between pages in the same site.

We assume pages with the same host-name are in the same site. Table 2 shows the number of nodes and edges after pruning in the UK data sets. 


\subsection{Results of Extracted Seeds using Proposed Algorithm}

We run our algorithm for seeds extraction, ExtractSeeds, on UK-2002 and UK-2005. This algorithm, as Figure 4 shows, sets CoverDensity to 100 for seeds extraction. It searches and extracts complete bipartite cores and then, at each step, selects the seeds from hub nodes in the bipartite sub-graph (see Figure 2). Figure 6 shows the size of extracted hubs and authorities in different iteration from UK-2002. It is clear that these cores are complete-bipartite. To reduce the impact of outlier hub sizes in the graphical presentation, we have used a log-log diagram. Figure 7 depicts the size of the extracted hubs and authorities in different iterations for UK2005.

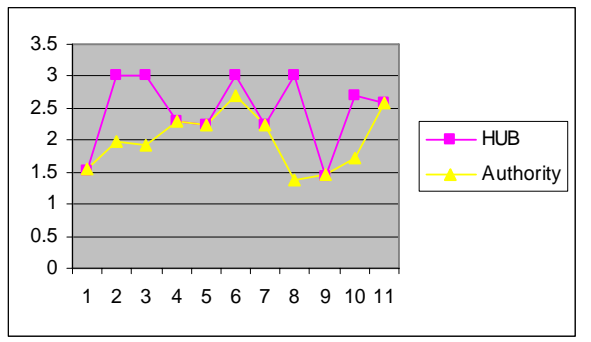

Figure 6. Log-Log diagram of Hub and Authority sizes Extracted from UK 2002 in different iteration.

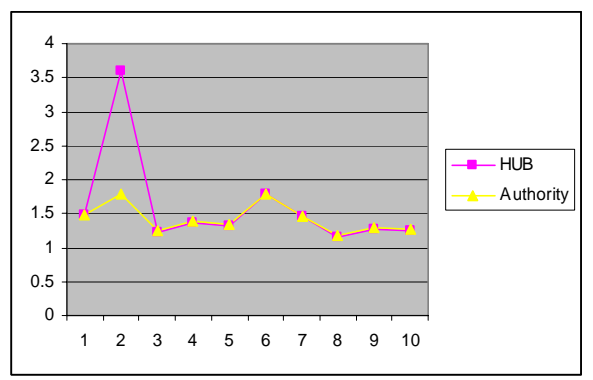

Figure 7. Log-Log diagram of Hub and Authority sizes Extracted from UK 2005 in different iteration

Normally, the hub sizes are bigger than the authority sizes. We obtained bipartite cores with very large hub sizes in UK-2002. So, we have limited the number of hubs to 999 in UK-2002 data set.

\subsection{Quality Analysis}

\subsubsection{Metrics for Analysis}

We used some different metrics to evaluate the quality of extracted seeds. The first metric is the distance between extracted seeds. As we have mentioned earlier, a crawler tends to extract web pages from different communities. Using HITSRanking and Iterative pruning we can conclude that extracted seeds are from different communities. To prove this, we measure the distances between extracted cores. We have defined core-distances as the nearest directed path between one of the nodes in the source core to one of the nodes in the destination core.

The second metric, is the PageRank of pages that will be crawled starting from these seeds. Previously, we have defined the most suitable pages in the web to be the pages with high PageRanks. Therefore, if the average PageRank of crawled pages, at each step of crawl, is bigger than a random crawl, especially at the beginning, then we can conclude that a crawl that starts from those seeds identified by our algorithm will result in better pages.

The third metric is the number of crawled pages at each step of crawling. We focus on a crawler whose goal is to download good pages in small iteration. Thus, if the number of crawled pages starting from extracted seeds by our method at each step is bigger than the number of crawled pages starting at random set, then we can conclude that our method leads a crawl toward visiting more pages in less iteration too.

For the first metric, we measure the distance between the cores. For the other two metrics, we need to crawl the graph starting from seeds extracted with our method and compare it with a crawl starting from randomly selected seeds.

\subsubsection{Result of Bipartite Core Distances}

We have measured the distance between all bipartite cores that were extracted from UK datasets and they had a reasonable distance in comparison with the diameter of the related graph. Figure 8 , shows the graphical representation of distances between 56 cores extracted from UK-2002. The number on top of each node indicates the iteration number in which the core has been extracted. Because the distance graph between nodes may have not an Euclidian representation, distances in this figure do not exactly match with real distances. The other important information is that bipartite cores in close iterations have a distance equal or bigger than average distance of related web graph. In addition, the cores that are close to each other (there is a short directed 
path between them) are identified in far iteration. As an example, the distance between core extracted from iteration 32 and the core extracted from iteration 47 is one. In this sample, the minimum distance between nodes is 1 and maximum distance is 13 . The average distance is 7.15 . As the diameter of UK-2002 data set is 14.9, core distances are fine.

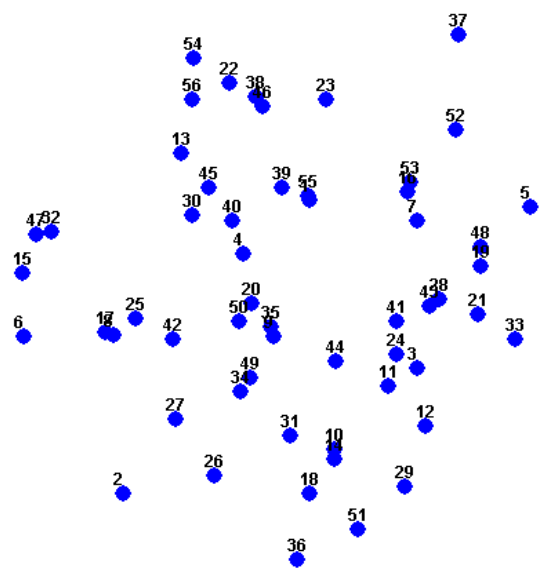

Figure 8. Graphical representation of distances between 56 extracted seeds from UK-2002 by our algorithm. The number on top of each node(core) indicates the iteration number in which the related node has been extracted.

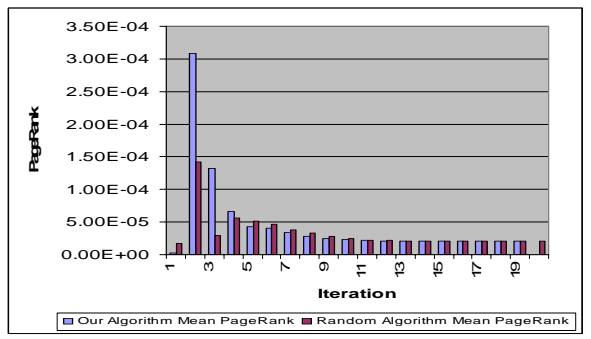

Figure 9. Comparison between PageRank of Crawled pages starting from 10 seeds extracted by our method on UK-2002 and Random Seeds.

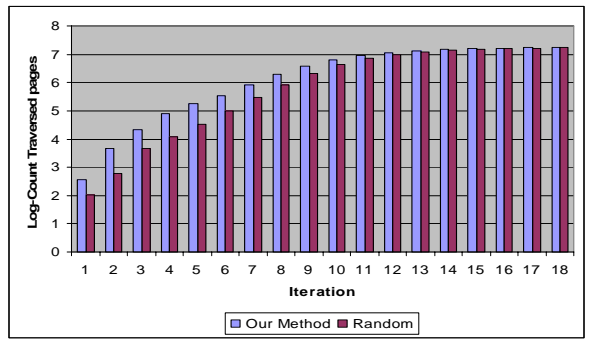

Figure 10. Comparison between Log Count diagram of pages visited at each Iteration starting from 10 seeds extracted by our method and 10 seeds selected randomly from UK-2002.

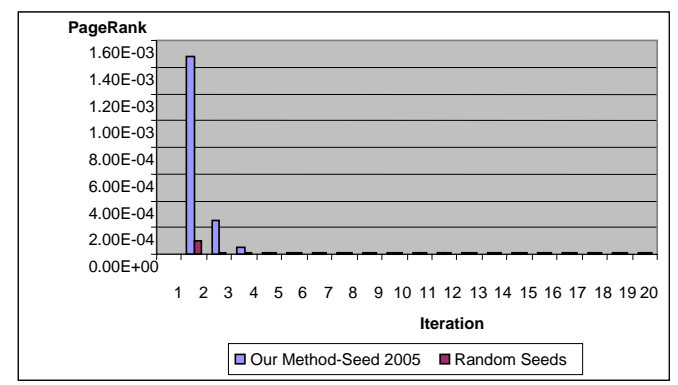

Figure 11. Comparison between PageRank of Crawled pages starting 10 seeds extracted from UK-2005 by our method and 10 Random seeds selected from UK-2005.

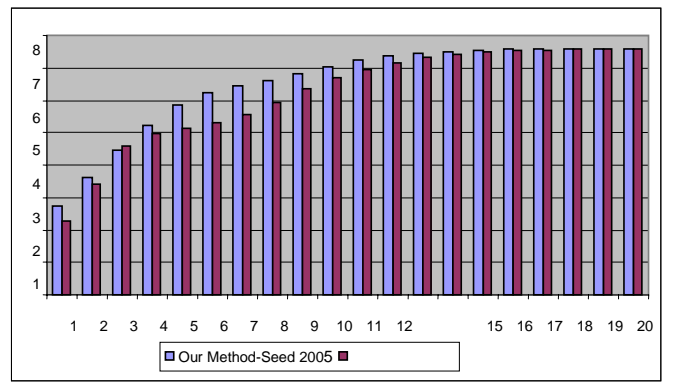

Figure 12. Comparison between Log Count Diagram of pages visited at each Iteration starting from 10 seeds extracted by our method from UK-2005 and 10 seeds selected randomly on UK-2005.

\subsubsection{Result of Average PageRank and Visit Count}

In this section, we evaluate the second and third metrics defined for evaluation. For UK-2002 we have executed the Extract-Seed algorithm with SeedCount $=10$. Therefore, the algorithm extracts one seed from each core in iteration. Then, we have started a crawling on UK-2002 data set implementing BFS strategy and measured the average PageRank of visited pages in each crawl depth, and the number of pages visited in each crawl depth. Then, we have compared the results with those gained from a crawl starting from random seeds for the same graph.

Figure 9 shows the comparison of average PageRank of crawl starting from seeds extracted with our method and a crawl starting from random seeds. Except the first depth (iteration) of crawl, in the other steps, up to step 4, the average PageRank of pages crawled with our method appear to be better. Specially, in the second and third iterations, the difference is superior. In the later iterations average PageRank of visited pages are close. 
Figure 10 shows the comparison of log-number of pages visited in each depth of crawl on UK-2002. For better graphical representation, we have computed log-count of visited pages. Apparently, the results of our method are always better than crawl starting random seeds and a crawl with seeds extracted with our method downloads more pages in less iteration. Figures 11 and 12 show the experiments on UK-2005. The same results appear here too.

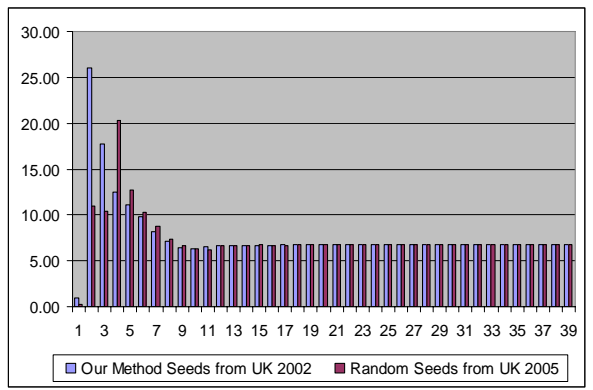

Figure 13. Comparison between PageRank of Crawled pages starting 11 seeds extracted from UK-2002 by our method and 11 Random seeds selected from UK-2005.

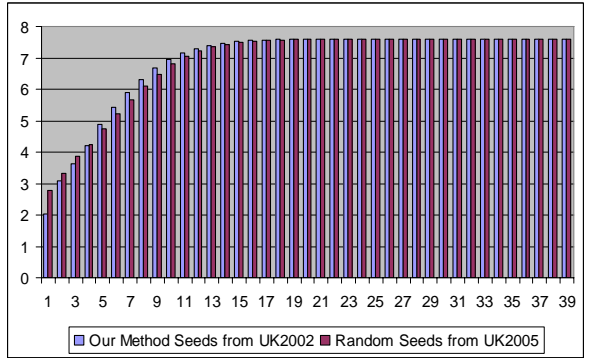

Figure 14. Comparison between Log Count Diagram of pages visited at each Iteration starting from 11 seeds extracted by our method from UK-2002 and 11 seeds selected randomly on UK-2005.

\subsubsection{Good Seeds for a New Crawl}

Using proposed algorithm we have discovered seeds from UK 2002 and UK 2005. Then we have evaluated the utility of these seeds using three evaluation criteria. These evaluations are good, but a real crawler has not access to seeds of a web graph which it is going to crawl. We should show that the result is always good if we start the crawl using seeds extracted from an old crawled graph.

In this section, we show the result of crawling on UK 2005 using seeds extracted by proposed algorithm and we compare it by randomly crawled seeds to simulate the real environment. Before algorithm's execution, we have checked the validity of seeds found from UK-2002 in UK-2005 data set. If a seed does not exist in a newer graph, then we remove that seed from our seeds set. Our experiments show that only 11 percent of seeds exist in the new data set. In fact, we have extracted 100 seeds from UK 2002 to be sure that we have 11 valid seeds in UK 2005.

Figure 13, shows the comparison of average PageRank of crawl starting from seeds extracted with our method and a crawl starting from random seeds. The result of our method is better until iteration 3. Figure 14, shows the comparison of lognumber of pages visited in each depth of the crawl. In this case, the result of our method is better than the random case between steps 4 and 15. In fact, our method download pages with high Page Rank till iteration 3 and next it crawls more pages than the random case till iteration 15 . After that, the result is nearly the same. Therefore, we can conclude that a crawler can download qualified web pages in less iteration; starting generated seeds set using our algorithm in less iteration.

\section{CONCLUSION AND FUTURE WORKS}

Crawlers like to download more good pages in less iteration. In this paper, we have presented a new fast algorithm with running time $O(n)$ for extracting seeds set from previously crawled web pages. In our experiments we have showed that if a crawler starts crawling from seeds set identified by our method, then it will crawl more pages with higher PageRank in less iteration and from different communities, than starting a random seeds set. In addition, we have measured the distance between selected seeds to be sure that our seeds set contains nodes from different communities. According to our knowledge, this is the first seeds extraction algorithm that is able to identify and extract seeds from different communities.

Our experiments were on graphs containing at most 39M nodes and 183M edges. This method can be experienced on larger graph in order to investigate the resulting quality on them too.

Another aspect where improvement may be possible is the implementation of the seeds that are not found in a new crawl. In our experiments, we have simply ignored nodes present in an older graph but not in 
the newer one. This aspect may be improved by finding similar nodes in the newer graph.

\section{ACKNOWLEDGEMENTS}

Authors would like to thank Mohammad Mahdian for his helpful and valuable comment on earlier draft of this work.

\section{REFERENCES}

[1] Henzinger, M. R., 2003. Algorithmic challenges in Web Search Engines. Internet Mathematics, vol. 1, no. 1 , pp. 115-123.

[2] Cho,J. Garcia-Molina, H. and Page, L., 1998. Efficient Crawling through URL ordering. In Proceedings of the 7th International World Wide Web Conference, April, pp.161-172.

[3] Najork, Wiener, J. L., 2001. Breadth-First Search Crawling Yields High-Quality Pages, Proceedings of the 10th international conference on World Wide Web,pp. 114-118.

[4] Brin , S. and Page, L., 1998. The anatomy of a largescale hypertextual Web search engine. Proceedings of the seventh international conference on World Wide Web 7, pp. 107 - 117.

[5] Kleinberg,J., Lawrence, S., 2001. The Structure of the Web. Science, vol. 294. no. 5548, pp. 1849 1850.

[6] Andrei Z. Broder, Ravi Kumar, Farzin Maghoul, Prabhakar Raghavan, Sridhar Rajagopalan, Raymie Stata, Andrew Tomkins, Janet L. Wiener, 2000. Graph structure in the Web. Computer Networks, pp. 309-320.

[7] Jon M. Kleinberg,J., 1999. Authoritative Sources in a Hyperlinked Environment. Proc. 9th ACM-SIAM Symposium on Discrete Algorithms, pp.604-632.

[8] Kumar,R., Raghavan,P., Rajagopalan,S., Tomkins,A., 1999. Trawling the Web for Emerging Cyber-Communities. Computer Networks, vol. 33, no. 11, pp.1481-1493.

[9] William Flake,G., Lawrence,S., Giles,L., Coetzee,F., 2002. Self-Organization and Identification of Web Communities. IEEE Computer, vol. 35, no. 3, pp. 66-71.

[10] Laboratory for Web Algorithmics, [Online], Available: http://law.dsi.unimi.it/ [19 Jan. 2007]

[11] Boldi,P., and Vigna,S., 2004. The WebGraph framework I: Compression techniques. In Proc. of the Thirteenth International World Wide Web Conference, pp. 595-601.

[12] Boldi, P., Codenotti, B., Santini, M., Vigna, S., 2004, UbiCrawler: A Scalable Fully Distributed Web Crawler, Journal of Software: Practice \& Experience, , vol 34, no 8, pp. 711-726.

[13] Albert, R. Jeong, H. Barabasi, A.L., 2000, 'A random Graph Model for massive graphs', $A C M$ symposium on the Theory and computing. 